\title{
Stabilization of Neoclassical Tearing Modes by rf Current
}

\author{
Xiaojing Wang1, Xiaodong Zhang², Qingquan $\mathrm{Yu}^{3}$, Yang Zhang², Sizheng Zhu' ${ }^{2}$, \\ Xiaoguang Wang², Bin $\mathrm{Wu}^{2}$
}

${ }^{1}$ University of Science and Technology of China, Hefei, China

${ }^{2}$ Institute of Plasma Physics, Chinese Academy of Sciences, Hefei, China

${ }^{3}$ Max-Planck-Institut für Plasmaphysik, Garching, Germany

Email: wxj1989@mail.ustc.edu.cn

How to cite this paper: Wang, X.J., Zhang, X.D., Yu, Q.Q., Zhang, Y., Zhu, S.Z., Wang, X.G. and $\mathrm{Wu}$, B. (2017) Stabilization of Neoclassical Tearing Modes by rf Current. World Journal of Engineering and Technology, 5, 99-104.

https://doi.org/10.4236/wjet.2017.54B011

Received: September 14, 2017

Accepted: October 9, 2017

Published: October 12, 2017

\begin{abstract}
Neoclassical tearing mode (NTM) can degrade plasma confinement or even cause disruptions in existing tokamaks. Stabilization of the $m / n=3 / 2$ NTMs by radio frequency (rf) current is investigated by the modified Rutherford equation (MRE) in this paper. In a range of parameters, the required rf current for mode stabilization is obtained, which is linearly proportional to the bootstrap current density for both modulated current drive (MCD) and non-modulated current drive (NMCD), linearly (quadratically) to the radial width of the rf current for MCD (NMCD), and quadratically to the radial deviation of the rf current from the rational surface for both MCD and NMCD.
\end{abstract}

\section{Keywords}

Neoclassical Tearing Mode (NTM), Radio Frequency (RF) Current, Modified Rutherford Equation (MRE)

\section{Introduction}

The neoclassical tearing mode (NTM) is expected to form a magnetic island structure which locally flattens the plasma pressure, hence losing the bootstrap current [1] [2] [3] [4]. Low mode number NTMs, especially the $m / n=3 / 2 \quad(m$ and $n$ are the poloidal and toroidal mode numbers)and $2 / 1$ modes, are observed to cause the confinement degradation and/or disruptions, limiting the achievable plasma $\beta$ value well below the predictions of ideal magnetohydrodynamic (MHD) calculations for positive magnetic shear [4]-[12].

In general, NTMs can be stabilized by compensating the perturbed bootstrap 
current or tailoring the plasma current density profile using local radio frequency (rf) current. The driven current should be in the direction along the equilibrium plasma current, to compensate the missing bootstrap current inside the island [8] [9] [10] [13] [14] [15] [16]. The stabilization of NTMs by rf current drive has been successfully carried out in tokamak experiments, e.g. on ASDEX-U, DIII-D, JT-60U [7] [8] [12] [13] [17]. The modified Rutherford equation (MRE), governing the time evolution of the nonlinear island, has been calculated by several authors [4] [15] [16] [18]. However, the predictions of the required rf current for mode stabilization are still in question and this is the purpose of this paper.

\section{Island Evolution Equation}

The MRE which describes the temporal evolution of the full width of a magnetic island $w$ can be written as follows [3] [4] [19]

$$
\frac{\tau_{r}}{r_{s}} \frac{d w}{d t}=\Delta_{0}^{\prime} r_{s}+\Delta_{b}^{\prime} r_{s}+\Delta_{c d}^{\prime} r_{s}
$$

in which $\tau_{r}=\frac{\mu_{0} r_{s}^{2}}{1.22 \eta}, r_{s}$ is the rational surface and $\eta$ is the plasma resistivity. $\Delta_{0}^{\prime}$ is the tearing mode stability index, defined as the logarithmic discontinuity in the radial derivative of the perturbed magnetic flux function $\psi$.In the vicinity of the rational surface the well-known "constant $\psi$ approximation" is utilized. The bootstrap current perturbation term can be written as

$$
\Delta_{b}^{\prime}=4.6 \sqrt{\varepsilon} \frac{2 \mu_{0}\left(-p^{\prime}\right)}{B_{\theta}^{2}} L_{q} \frac{w}{w^{2}+w_{d}^{2}}
$$

where $B_{\theta}$ is the poloidal magnetic field, $p$ the plasma pressure, $\left.L_{q} \equiv \frac{q}{q^{\prime}}\right|_{r=r_{s}}$ the magnetic shear length at the rational surface, $q$ the safety factor, and $w_{d}$ is the characteristic island width above which the plasma pressure is flattened across the island. The prime denotes the radial derivative.

Equation (1) is obtained through averaging of the parallel projection of the Ohm's law over helical flux surfaces in the vicinity of the island

$$
E_{\|}=\eta\left(\delta j_{\|}-\delta j_{b}-\delta j_{c d}\right)
$$

It is convenient to introduce the normalized perturbed flux function [3] [4] [15]

$$
\Omega=\frac{\chi}{\psi}=8 \frac{x^{2}}{w^{2}}-\cos (h)
$$

with $\Omega=-1$ at the island O-point and $\Omega=1$ at the X-point, where $x=r-r_{s}$ and $h=m \theta+n \xi$ is the helical angle. Following Ref. [3], the flux surface average operator is defined as

$$
<F(\sigma, \Omega, h)>=\oint \frac{F(\sigma, \Omega, h)}{\sqrt{\Omega+\cos (h)}} d h
$$


for $\Omega>1$, and

$$
<F(\sigma, \Omega, h)>=\int_{-h_{\Omega}}^{h_{\Omega}} \frac{\frac{1}{2}(F(\sigma, \Omega, h)+F(-\sigma, \Omega, h))}{\sqrt{\Omega+\cos (h)}} d h
$$

for $\Omega \leq 1$. Here, $\sigma=\operatorname{sgn}(x), h_{\Omega}=\cos ^{-1}(-\Omega)$, and $F$ is a general function.

In this way, the driven current term is given by

$$
\Delta_{c d}^{\prime}=-\frac{16}{\pi} \frac{\mu_{0} I_{c d} L_{q}}{r_{s} B_{\theta}} \frac{1}{\left(2 w_{c d}\right)^{2}} \eta_{c d}
$$

where $I_{c d}$ is the magnitude of the rf driven current and $w_{c d}$ is the half width of the driven current. With a Gaussian profile for the driven current by rf waves [9] [15]

$$
j_{c d}=j_{c d 0} \exp \left[-\left(\frac{r-r_{c d}}{w_{c d}}\right)^{2}\right] \Pi\left(h_{0}, \Delta h\right)
$$

the stabilization efficiency $\eta_{c d}$ is given by

$$
\eta_{c d}=\frac{\left(2 w_{c d}\right)^{2}}{w^{2}} \frac{\int_{-1}^{+\infty} \frac{<\cos (h)>}{<1>}<j_{c d}>d \Omega}{\int_{-1}^{+\infty}<j_{c d}>d \Omega}
$$

where $j_{c d 0}$ is the peak amplitude of the driven current and the square box function $\Pi\left(h_{0}, \Delta h\right)$ is equal to 1 for $\left|h-h_{0}\right|<\Delta h$ and to 0 otherwise, defining the helical angle of the $\mathrm{rf}$ current deposition, where $h_{0}=\omega t, \omega$ is the mode rotation frequency, and $\Delta h$ is the instantaneous wave deposition width along the helical angle.

In this paper, we neglect the modification of the equilibrium plasma current profile by the current drive and the reduced island width, which can modify the $\Delta_{0}^{\prime}$.

\section{Required rf Current for Mode Stabilization}

In this paper the inverse aspect ratio $\epsilon \equiv a / R=0.28$ is utilized, where $a$ is the plasma minor radius and $R$ the major radius. Results obtained from the MRE utilizing Equation (1) for the $m / n=3 / 2$ magnetic island and its stabilization with rf current are shown in Figure 1, where $j_{b} / j_{p}=0.173, L_{q} / a=0.41$ at $r_{s} / a=0.555$ and $w_{c d} / a=0.05$ are taken. $\Delta_{0}^{\prime}=-m / r_{s}$ is assumed. The solid curve is the case without the driven current term. The dashed and the dot-dashed curves are for modulated current drive (MCD, 50\% on-time) with $I_{c d} / I_{p}=0.017$ and for non-modulated current drive (NMCD) with $I_{c d} / I_{p}=0.03$, respectively. For these values of $I_{c d} / I_{p}$ the maximal of $d w / d t$ is zero, i.e., the required driven current for mode stabilization.

The required $I_{c d} / I_{p}$ for mode stabilization is shown as a function of $j_{b} / j_{p}$ for $w_{c d} / a=0.05$ in Figure 2. The solid (dashed) curve is for MCD (NMCD). It is seen the required $I_{c d} / I_{p}$ depends linearly on $j_{b} / j_{p}$ for both MCD and NMCD. 


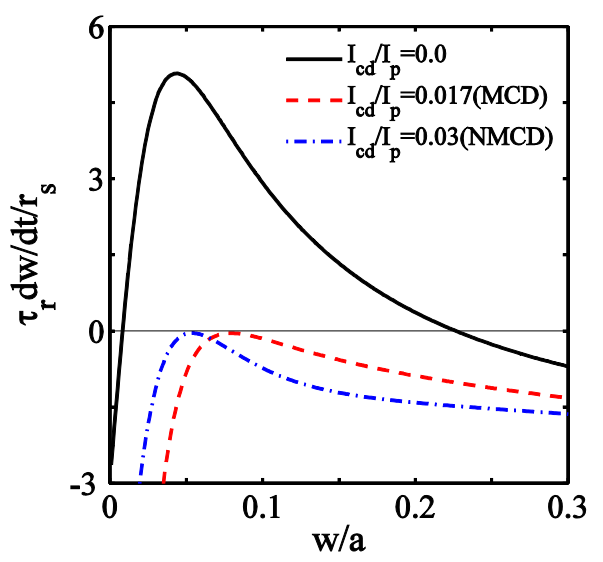

Figure 1. Evaluation of $d w / d t$ from MRE for the $m / n=3 / 2$ magnetic island with $j_{b} / j_{p}=0.173, L_{q} / a=0.41$ at $r_{s} / a=0.555$ and $w_{c d} / a=0.05 . \Delta_{0}^{\prime}=-m / r_{s}$ is utilized. The solid curve is the case without the driven current. The dashed and dot-dashed curves are for MCD with $I_{c d} / I_{p}=0.017$ and for NMCD with $I_{c d} / I_{p}=0.03$, respectively.

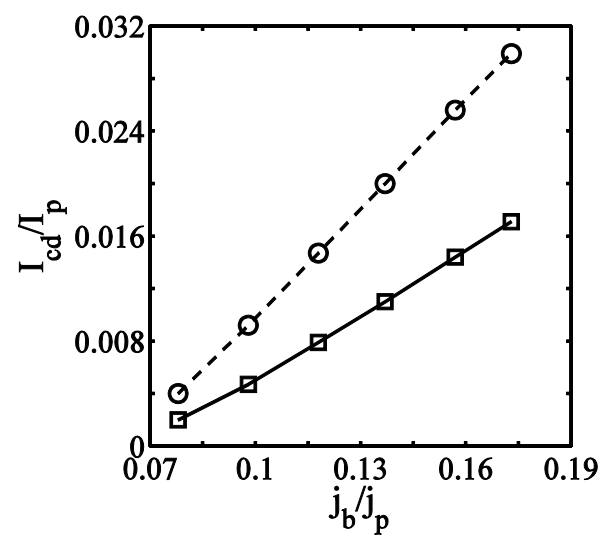

Figure 2. Required $I_{c d} / I_{p}$ for mode stabilization versus $j_{b} / j_{p}$ for $w_{c d} / a=0.05$. The solid (dashed) curve is for MCD (NMCD).

Figure 3 shows the ratio $I_{c d} / I_{p}$ as a function of the half width of driven current $w_{c d}$ for $j_{b} / j_{p}=0.173$. There is an approximate linear and a quadratic relationship for MCD and NMCD, respectively. According to Equations (1) and (7) the required driven current is proportional to $y$, where $y=\left(\frac{w_{c d}}{a}\right)^{2} \frac{1}{\eta_{c d}}$. For different island width there is always a linear (quadratic) relationship between $y$ and $w_{c d}$ with MCD (NMCD).

For above results the localized current drive is applied right at the resonant surface. The radial misalignment between the rf waves deposition and the resonant surface significantly affects the NTM stabilization. For $w_{c d} / a=0.05$ and $j_{b} / j_{p}=0.173$, the required $I_{c d} / I_{p}$ for mode stabilization is shown in Figure 4 as a function of the normalized radial deviation of $\mathrm{rf}$ current deposition from the rational surface, $x_{c d}=\frac{r_{c d}-r_{s}}{a}$, for MCD (solid curve) and NMCD (dashed). It is 


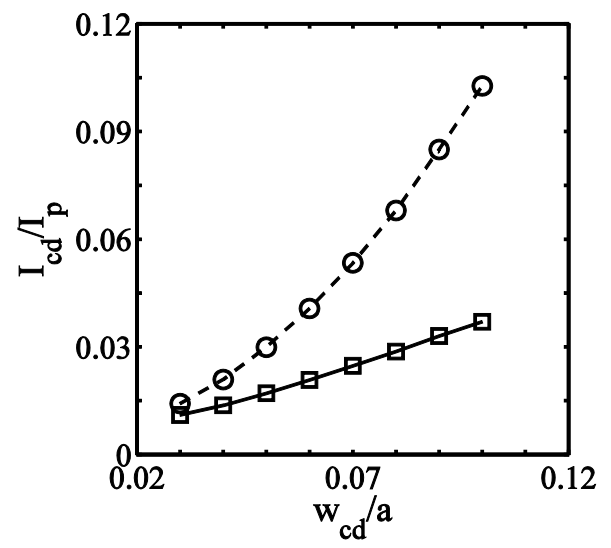

Figure 3. Required $I_{c d} / I_{p}$ for mode stabilization versus $w_{c d} / a$ for $j_{b} / j_{p}=0.173$. The solid (dashed) curve is for MCD (NMCD).

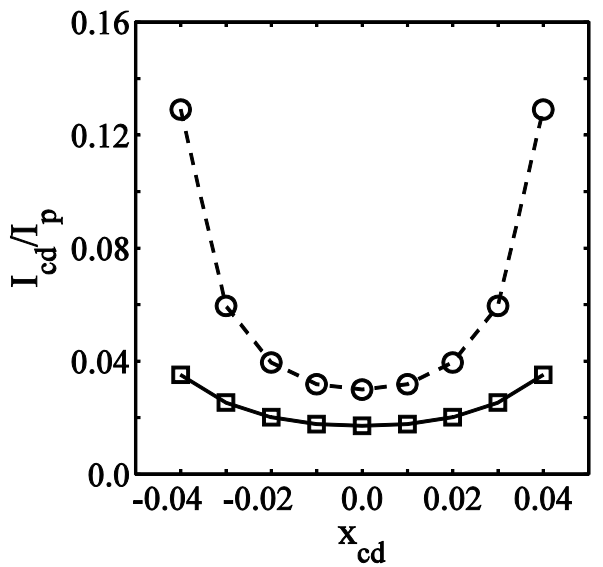

Figure 4. Required $I_{c d} / I_{p}$ for mode stabilization versus the radial deviation of the $\mathrm{rf}$ current $x_{c d}$, where $x_{c d}=\frac{r_{c d}-r_{s}}{a}$. The solid (dashed) curve is for MCD (NMCD).

seen between the ratio $I_{c d} / I_{p}$ and the radial deviation $X_{c d}$ there is always an approximate quadratic relationship for both MCD and NMCD. A shift of rf current deposition away from the rational surface greatly decreases the stabilizing efficiency, since the applied driven current is not around the island's o-point. The required driven current for NMCD is much more than that for MCD when $\left|x_{c d}\right|>0.03$. The reason is that NMCD deposited around the island separatrix and the island $\mathrm{X}$-point is destabilizing.

\section{Conclusions}

The magnetic island width changes with the pressure profile, which in turn affects the island growth. The modification of the equilibrium plasma current profile by the current drive can affect the $\Delta_{0}^{\prime}$. These are not considered in our calculations. And the numerical simulation on the required driven current for mode stabilization which consistently calculates the pressure profile and the plasma current density profile will be the future work. 
In summary, the required driven current for NTMs' stabilization by rf current is investigated by the MRE. The simple linear and/or quadratic relations are found between the required rf current for mode stabilization and the bootstrap current density, the radial width and radial deviation of the rf current from the rational surface for both MCD and NMCD.

\section{Acknowledgements}

This work was supported by the National Magnetic Confinement Fusion Science Program of China (Contract No. 2012GB103000 and 2012GB103003) and the National Natural Science Foundation of China (Grant No. 11475225).

\section{References}

[1] Carrera, R., et al. (1986) Phys. Fluids, 29, 899. https://doi.org/10.1063/1.865682

[2] Hegna, C.C. and Callen, J.D. (1992) Phys. Fluids B, 4, 1855. https://doi.org/10.1063/1.860039

[3] Fitzpatrick, R. (1995) Phys. Plasmas, 2, 825. https://doi.org/10.1063/1.871434

[4] Hegna, C.C. and Callen, J.D. (1997) Phys. Plasmas, 4, 2940. https://doi.org/10.1063/1.872426

[5] Sing, D.C., et al. (1993) Phys. Fluids B, 5, 3239. https://doi.org/10.1063/1.860659

[6] Classen, I.G.J., et al. (2007) Phys. Rev. Lett., 98, Article ID: 035001 https://doi.org/10.1103/PhysRevLett.98.035001

[7] Gantenbein. G., et al. (2000) Phys. Rev. Lett., 85, 1242. https://doi.org/10.1103/PhysRevLett.85.1242

[8] Zohm, H., et al. (1999) Nucl. Fusion, 39, 577. https://doi.org/10.1088/0029-5515/39/5/101

[9] Yu, Q., et al. (2000) Phys. Plasmas, 7, 312. https://doi.org/10.1063/1.873799

[10] Yu, Q., et al. (2004) Phys. Plasmas, 11, 1960. https://doi.org/10.1063/1.1710521

[11] Yu, Q. and Günter, S. (2008) Nucl. Fusion, 48, Article ID: 065004.

[12] Maraschek, M., et al. (2007) Phys. Rev. Lett., 98, Article ID: 025005. https://doi.org/10.1103/PhysRevLett.98.025005

[13] Isayama, A., et al. (2000) Plasma Phys. Control. Fusion, 42, L37. https://doi.org/10.1088/0741-3335/42/12/102

[14] La Haye, R.J. (2006) Phys. Plasmas, 13, Article ID: 055501. https://doi.org/10.1063/1.2180747

[15] Giruzzi, G., et al. (1999) Nucl. Fusion, 39, 107. https://doi.org/10.1088/0029-5515/39/1/307

[16] Perkins, F.W., et al (1997) Proceedings of the 24th EPS Conference on Controlled Fusion and Plasma Physics (European Physical Society), Berchtesgaden, 9-13 June 1997, 1017.

[17] La Haye, R.J., et al. (2002) Phys. Plasmas, 9, 2051. https://doi.org/10.1063/1.1456066

[18] Zohm, H. (1997) Phys. Plasmas, 4, 3433. https://doi.org/10.1063/1.872487

[19] Sauter, O., et al. (2002) Plasma Phys. Control. Fusion, 44, 1999. https://doi.org/10.1088/0741-3335/44/9/315 